\title{
Aeromonas spp.: an emerging pathogen?
}

\author{
Andrea Bartolini, ${ }^{1,2}$ Giulia Zorzi, ${ }^{1,2}$ Elena Castellani, ${ }^{1,2}$ Valeria Besutti ${ }^{1}$ \\ ${ }^{1}$ Operative Unit of Microbioly and Virology, Hospital of Padua; ${ }^{2}$ Department of Molecular \\ Medicine, University of Padua, Italy
}

\section{Summary}

The aim of this study is to identify and monitor the presence of Aeromonas spp. strains in stool cultures. We analyzed 5564 stool cultures from September 2012 to August 2013. Sixty-three patients were positive for Aeromonas spp. The most frequent symptoms were: diarrhea (46.0\%) and abdominal pain (12.7\%). Pediatric subjects were 28. Samples' microscopic examination showed leukocytes in $38.1 \%$ of cases. It is still controversial whether Aeromonas are responsible for human gastroenteritis, but their presence in faecies of symptomatic patients supports their etiologic role. We propose search for toxins by polymerase chain reaction to identify strains that require an antibiotic therapy.

\section{Introduction}

Aeromonas spp. are Gram-negative rods, facultative anaerobic, nonspore-forming, ubiquitous bacteria $(2,3,4)$. Aeromonas spp. strains have been isolated in different aquatic environments including treated drinking water, in the soil and in many foods, including meat and milk $(2,6)$. The genus Aeromonas shares many biochemical features with Enterobacteriaceae, from which it can be differentiated by the oxidase

Correspondence: Andrea Bartolini, Servizio di Microbiologia e Virologia, Azienda 0spedaliera di Padova, Via Giustiniani 2, 35128 Padova, Italy. Tel.: +39.049.821305 - Fax: +39.049 .8213054$

E-mail: andbarto@libero.it

Key words: Aeromonas spp., diarrhea, emerging waterborne gastroenteritis.

Fundings: this work was supported by the University of Padua.

Contributions: the authors contributed equally.

Conflict of interest: the authors declare no potential conflict of interest.

Received for publication: 18 August 2014.

Accepted for publication: 29 May 2015.

(C) Copyright A. Bartolini et al., 2015

Licensee PAGEPress, Italy

Microbiologia Medica 2015; 30:4674

doi:10.4081/mm.2015.4674

This article is distributed under the terms of the Creative Commons Attribution Noncommercial License (by-nc 3.0) which permits any noncommercial use, distribution, and reproduction in any medium, provided the original author(s) and source are credited. test, positive for Aeromonas $(2,4)$. The genus includes several species, including the mesophilic Aeromonas hydrophila, Aeromonas caviae, Aeromonas sobria, Aeromonas veronii, Aeromonas schubertii and psychrophilic Aeromonas salmonicida, forced fishes' pathogen, not correlated with human disease $(2,3,4,6)$. The mesophilic species have shown to possess several virulence factors (adhesins, hemolysins, cytotonic and cytotoxic enterotoxins), all of them probably involved in human disease $(2,4)$. Primary toxins produced are hemolysins, among which the most significant is the aerolysin expressed by many strains of $A$. hydrophila and A. sobria. Furthermore it has been demonstrated the presence of at least one citotonic toxin with activity similar to cholera toxin (5). Aeromonas spp. strains can cause gastroenteritis, skin and soft tissue infections and bacteremia in immunocompromised patients $(2,4)$. In particular A. hydrophila was associated with two distinct types of gastroenteritis: a cholera-like form with watery diarrhea and a dysenteric form $(2,4)$. Several factors, including patient's age, state of immunocompetence, presence of concomitant diseases, infectious load and virulence factors expression affect the pathogenicity of Aeromonas spp. (5). Many studies have found an increase of Aeromonas spp. isolation from fecal samples during the hottest months of the year, an event that can reasonably be attributed to the fact that the mesophilic species grow optimally at high temperatures, thereby leading to an increase in the concentration of bacteria in freshwater ecosystems and in home drinking water (3). Gastrointestinal infections caused by Aeromonas spp. are generally self-limiting and antibiotic therapy is usually used in cases of severe and not responsive infections $(2,4)$.

Aeromonas spp. strains are universally considered resistant to penicillins, due to inducible betalactamase production, while usually show themselves sensible to aminoglycosides, tetracyclines, chloramphenicol, trimethoprim-sulfamethoxazole and quinolones. They are also sensible to second and third cephalosporins generation $(2,4)$. Aeromonas spp. strains are commonly isolated from fecal samples of children aged less than 5 years (2). It was shown that the number of cases of infection by Aeromonas may be underestimated because of sample's inadequate management, both in preanalytic phase than in the culture method (4). The purpose of this study is to identify and monitor the presence of such strains in stool cultures of patients attending the operative unit of Microbiology and Virology of Padua.

\section{Materials and Methods}

The analysis was carried out in the period between September 2012 and August 2013 on the 5564 stool culture requests from Hospital of Padua departments and clinics and by external users related to our structure. The samples were collected in tubes with screw cap containing Cary-Blair transport medium modified (FecalSwab ${ }^{\mathrm{TM}}$, Brescia, Italy) and then they were inoculated with the automated WASP ${ }^{\circledR} \mathrm{BD}^{\mathrm{TM}}$ 
(Becton, Dickinson, Franklin Lakes, NJ, USA) on Aeromonas/Yersinia Agar, selective differential medium. This medium is a variation of CIN agar (agar cefsulodin-irgasan-novobiocin) that not only promotes the growth of Yersinia enterocolitica but also of Aeromonas spp., because of a lower content of cefsulodin (1). The plates were incubated under aerobic conditions at a temperature of $25 \pm 2{ }^{\circ} \mathrm{C}$ for 48 hours. Aeromonas spp. strains produce light colonies with pink to red center, similar to Yersinia. On such media other Enterobacteriaceae are able to grow, such as Citrobacter and Serratia, not always distinguishable on the basis of colony morphology. For strain's identification we used BioMerieux Vitek ${ }^{\circledR} 2$ andVitek ${ }^{\circledR}$ MS, while for the antibiogram we used BioMerieux Vitek ${ }^{\circledR} 2$ (BioMerieux, Marcy-l'Étoile, France).

\section{Results}

In our records 63 patients were positive for Aeromonas spp., and more precisely 55 for $A$. hydrophila and 8 for A. sobria. Among these patients, 28 were male (44.4\%) and 35 were females (55.6\%). It is important to note that the paediatric subjects (aged less than 14 years) were $28(44.4 \%)$ and in particular those aged less than 3 years accounted for $31.3 \%$ of the total. The foreigners were 9 patients (14.3\%). From the data that we have collected from the medical history card, when available, and from interviews with the patients or the doctors, the symptoms that we found most frequently was distributed as follows: 29 patients reported diarrhea (46.0\%); 8 patients complained of abdominal pain (12.7\%).

As for the 26 remaining patients, representing the $41.3 \%$, unfortunately no anamnestic information was available whatsoever, whilst the coproculture screening was carried out for other unspecified purposes.

In 9 cases we found a co-infection with other intestinal pathogens, in detail: 4 Campylobacter spp. (culture method); 2 Yersinia enterocolitica (culture method); 1 Salmonella spp. (culture method); 1 Clostridium difficile (rapid enzyme immunoassay); 1 Rotavirus (rapid enzyme immunoassay).

As a further study we carried out a microscopic examination of the samples and we detected the significant presence of leukocytes in $38.1 \%$ of cases.

As for the antibiogram we tested the following molecules: ampicillin, amoxicillin/clavulanic acid, piperacillin/tazobactam, cefotaxime, cefepime, ciprofloxacin, and gentamicin. All strains were resistant to ampicillin and amoxicillin/clavulanic acid; 12 strains were also resist- ant to piperacillin/tazobactam, 1 strain was resistant to cefotaxime and 1 strain showed resistance to gentamicin.

\section{Discussion and Conclusions}

It is still controversial whether Aeromonas spp. strains are responsible for human gastroenteritis, but their presence in faeces of symptomatic patients, in the absence of other known pathogens, supports their etiologic role. Aeromonas ubiquitous nature in aquatic environments makes it possible the interaction with humans continuous and inevitable. It is therefore conceivable that some Aeromonas spp. strains, presenting the right set of virulence factors, are likely to induce gastrointestinal diseases.

In our situation, and in selected cases guided by a proper medical history, we propose to combine the culture method with PCR research of specific toxins of Aeromonas spp. to identify pathogenic strains with the aim of a precise etiological diagnosis as well as for a contingent antibiotic treatment.

\section{References}

1. Abbott SL. Aeromonas. In: Manual of clinical microbiology. $8^{\text {th }}$ ed. Murray PR, Baron EJ, Jorgensen JH, et al, eds. Washington DC: American Society for Microbiology; 2003. pp 684-701.

2. Igbinosa IH, Igumbor EU, Aghdasi F, et al. Emerging Aeromonas species infections and their significance in public health. ScientificWorldJournal 2012; 2012: 625023.

3. Janda JM, Abbott SL. The genus Aeromonas: taxonomy, pathogenicity, infection. Clin Microbiol Rev 2010; 23: 35-73.

4. Parker JL, Shaw JG. Aeromonas spp. Clinical microbiology and disease. J Infect 2011; 62: 109-118.

5. World Health Organization. Addendum: microbiological agents in drinking water. Guidelines for drinking-water quality, second edition. 2002. Available frm: http://www.who.int/water_sanitation_ health/dwq/admicrob1.pdf

6. World Health Organization. Guidelines for drinking-water quality, fourth edition. 2011. Available from: http:/www.who.int/water_sanitation_health/publications/2011/dwq_guidelines/en/ 